\title{
Combined oropharyngeal/nasal swab is equivalent to nasopharyngeal sampling for SARS-CoV-2 diagnostic PCR
}

Tania Desmet ${ }^{1}$, Peter De Paepe ${ }^{1}$, Jerina Boelens ${ }^{2}$, Liselotte Coorevits ${ }^{2}$, Elizaveta Padalko ${ }^{2}$, Stien Vandendriessche ${ }^{2}$, Isabel Leroux-Roels ${ }^{2,3}$, Annelies Aerssens ${ }^{3}$, Steven Callens ${ }^{4}$, Eva Van Braeckel ${ }^{5}$, Thomas Malfait ${ }^{5}$, Frank Vermassen ${ }^{6}$ and Bruno Verhasselt $2^{2 *}$

\begin{abstract}
Background: Early 2020, a COVID-19 epidemic became a public health emergency of international concern. To address this pandemic broad testing with an easy, comfortable and reliable testing method is of utmost concern. Nasopharyngeal (NP) swab sampling is the reference method though hampered by international supply shortages. A new oropharyngeal/nasal (OP/N) sampling method was investigated using the more readily available throat swab.

Results: 35 patients were diagnosed with SARS-CoV-2 by means of either NP or OP/N sampling. The paired swabs were both positive in 31 patients. The one patient who tested negative on both NP and OP/N swab on admission, was ultimately diagnosed on bronchoalveolar lavage fluid. A strong correlation was found between the viral RNA loads of the paired swabs $(r=0.76 ; P<0.05)$. The sensitivity of NP and OP/N analysis in hospitalized patients $(n=28)$ was $89.3 \%$ and $92.7 \%$ respectively.
\end{abstract}

Conclusions: This study demonstrates equivalence of NP and OP/N sampling for detection of SARS-CoV-2 by means of rRT-PCR. Sensitivity of both NP and OP/N sampling is very high in hospitalized patients.

Keywords: Combined oropharyngeal/nasal swab, SARS-CoV-2, nasopharyngeal swab, rRT-PCR, COVID-19.

\section{Background}

A pandemic of respiratory disease caused by SARS-CoV2 began in Wuhan, China in December 2019 and quickly spread to every continent. On February 1st, 2020, the disease was declared a Public Health Emergency of International Concern (PHEIC) by the World Health Organization (WHO). [1] By end 2020, over 80 million people were infected and almost 1,800,000 died.

Real-time reverse transcriptase-polymerase chain reaction (rRT-PCR) on nasopharyngeal swab material is

\footnotetext{
* Correspondence: Bruno.verhasselt@uzgent.be

${ }^{2}$ Department of Medical Microbiology, Ghent University Hospital, 9000 Ghent, Belgium

Full list of author information is available at the end of the article
}

typically used to confirm the clinical diagnosis. $[1,2]$ Nasopharyngeal (NP) swab remains the reference sampling method, while recent studies suggest that nasal swab and saliva sampling may be nearly equivalent. [36] The detection of SARS-CoV-2 in oropharyngeal swabs seems less sensitive. [7-12]

In order to address a pandemic, extensive mapping and therefore broad testing with an easy and comfortable sampling method is of utmost importance. In the context of an international shortage in nasopharyngeal swabs, the search for a suitable alternative is a global health priority. This study evaluates a new combined oropharyngeal/nasal $(\mathrm{OP} / \mathrm{N})$ sampling method using the more readily available throat swab. 


\section{Results}

During the study period, 41 SARS-CoV-2 PCR-confirmed patients were diagnosed at Ghent University Hospital. Five patients were excluded because no $\mathrm{OP} / \mathrm{N}$ swab was sampled upon admission. Seventy-five samples from the 36 remaining patients were analysed. All patients except two had 2 samples ( $1 \mathrm{NP}$ and $1 \mathrm{OP} / \mathrm{N})$, one patient had 3 samples $(2 \mathrm{NP}, 1 \mathrm{OP} / \mathrm{N})$, one patient had 4 samples $(2 \mathrm{NP}, 1$ $\mathrm{OP} / \mathrm{N}, 1 \mathrm{BAL}$ ). The median age was 61 years (range, 2290), 21 out of 36 patients (58\%) were male and 28 (78\%) patients were hospitalized. Patients were grouped for severity of illness according to the $\mathrm{NIH}$ guidelines: 7/36 showed mild illness (19\%), 10/36 showed moderate illness (28\%), $13 / 36$ showed severe illness (36\%) and 5/36 were critical ill (14\%). One patient was presymptomatic (3\%). Overall, 35/ 36 patients were diagnosed with SARS-CoV-2 by means of either NP or $\mathrm{OP} / \mathrm{N}$ sampling on admission. The paired swabs were both positive in 31 patients. In two patients, NP swabs were positive (Ct 36.47 and 38.38) with negative OP/ $\mathrm{N}$ swabs. In two other patients, $\mathrm{OP} / \mathrm{N}$ swabs were positive (Ct 34.14 and 41.39) with negative NP swabs. The one patient who tested negative on both NP and OP/N swab at admission, was ultimately diagnosed on BAL fluid (Ct 35.80). There was no difference in $\mathrm{Ct}$ value, both for $\mathrm{OP} / \mathrm{N}$ and NP sampling, among the groups with different severity of illness ( $P=0.459$ and $P=0.231$, respectively). The 5 patients $(5 / 36)$, in whom the paired swabs were not both positive, belonged to different groups of disease severity (1 mild, 1 moderate, 2 severe, 1 critical ill).

When the paired OP/N and NP samples were compared in the 31 patients who tested positive in both, the median Ct for SARS-CoV-2 PCR was not significantly different: 27.5 cycles (range, 15.7-40.4) in NP samples vs. $27.2 \mathrm{cy}$ cles (range, 14.1-40.2) $(P=0.576)$ in OP/N samples.

Besides, a strong correlation was found between the $\mathrm{Ct}$ values of the paired swabs $(r=0.76 ; P<0.05)$ (Fig. 1). In accordance, quantification of human DNA by real-time PCR did not show a significant difference in yield between both methods (data not shown). Of the 28 hospitalized patients, 27 were diagnosed by means of either NP (25) or OP/N (26) sampling. Thus, when considering a PCR positive result on BAL fluid as the ultimate confirmation of COVID-19 diagnosis, sensitivity of NP and $\mathrm{OP} / \mathrm{N}$ analysis were $89.3 \%$ and $92.7 \%$ respectively in hospitalized patients $(n=28)$.

\section{Discussion}

Nasopharyngeal swab is considered to be the reference sampling method in suspected COVID-19 patients. [3,

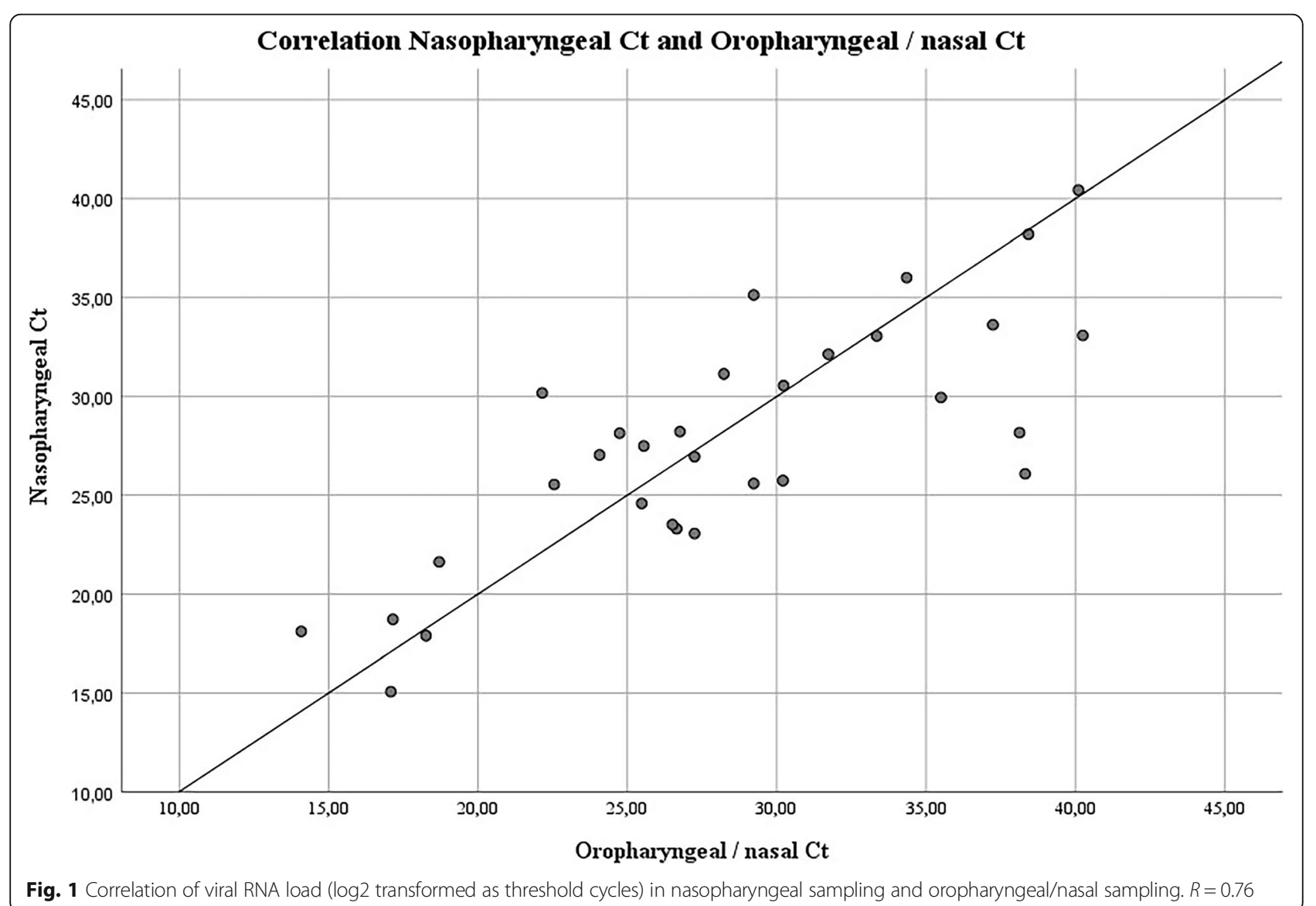


4] In the context of an international shortage in nasopharyngeal swabs, the search for a suitable alternative is a burning issue. Moreover, given the need of extensive population testing, an easy and patient-friendly sampling method is preferred. Salivary sampling has been reported as a reliable tool to detect SARS-CoV-2 in COVID-19 patients. This study, however, did only include patients with severe to very severe disease and did not provide information on mildly or moderately ill patients. [6] Nasal sampling was proposed as an alternative to nasopharyngeal sampling, though appears to be inferior to nasopharyngeal sampling (9.1\% false negatives). [5] Oropharyngeal (OP) sampling is less sensitive (compared both to nasopharyngeal and nasal sampling) according to several studies. Wang X. et al. (2020) and Wang W. et al. (2020) reported a positivity rate of OP sampling of $27 \%$ and $32 \%$ respectively in COVID-19 patients. Sensitivity results were not reported. $[8,11]$

To our knowledge, our study is the first to show that the molecular detection of SARS-CoV-2 on combined $\mathrm{OP} / \mathrm{N}$ swabs was non-inferior to nasopharyngeal sampling. [3, 4] We found a high correlation between the viral RNA loads of the paired $\mathrm{OP} / \mathrm{N}$ and NP samples. Besides, the sensitivities of both NP and OP/N sampling in hospitalized patients were $89.3 \%$ and $92.7 \%$ respectively. Large differences in sensitivity of NP sampling were published ranging from $63-73.3 \%$. [10, 11, 13] It should be mentioned however that, to our knowledge, no peer-reviewed studies or large trials regarding this subject are available. The gold standard to which tests under investigation are to be compared, is not clear and differs among various publications, although consensus seems to exist about the positioning of BAL fluid as the most reliable testing method. [10]

\section{Conclusion}

This study, performed in symptomatic patients with a broad age range and various degrees of disease severity, demonstrates equivalence of NP and OP/N sampling for detection of SARS-CoV-2 by means of rRT-PCR. Sensitivity of both NP and OP/N sampling is very high in hospitalized patients. These results warrant further evaluation of the combined oropharyngeal/nasal testing strategy in pauci- or asymptomatic individuals in order to tackle the global shortage in NP swabs. In this context, the new sampling strategy described here was used by the Belgian Government to guide a national screening campaign in residential care centres.

\section{Methods}

This prospective observational study was conducted at Ghent University Hospital, Belgium from April 7th to May 1st, 2020. Upon presentation at the emergency department, all patients with suspected COVID-19 (based on respiratory and inflammatory symptoms) in whom a NP test was indicated for diagnosis, were candidates for a combined $\mathrm{OP} / \mathrm{N}$ swab, sampled according to a specific protocol, developed in Ghent University Hospital. At inclusion, no distinction was made based upon disease severity or the need for hospitalization. Hospitalised patients with highly suspect clinical and radiological features of COVID-19 and negative PCR-sample on admission were retested with an additional NP and/or anal swab and finally, if still tested negative, on bronchoalveolar lavage (BAL) fluid for definite PCR-diagnosis. In ambulatory patients no retesting was performed. Patients were grouped for severity of illness according to the NIH guidelines. [14]

Specimens from $\mathrm{OP} / \mathrm{N}$ were obtained by rubbing the oropharyngeal space twice at both sides of the uvula and placing the same swab into both nasal cavities until a slight resistance was felt (supposed midturbinate). NP sampling was performed after $\mathrm{OP} / \mathrm{N}$ sampling in order not to 'contaminate' the midturbinate part of the nose by viral material from the nasopharynx as may be expected when performed in reversed order. Each swab was rotated three complete turns for optimal mucosal contact. For NP sampling a flexible mini tip flocked swab (in Amies transport medium or in universal transport medium (UTM)) was used (Cat. numbers $481 \mathrm{CE}$ and $305 \mathrm{C}$ respectively, $\mathrm{Copan}^{\circ}$, Italy). OP/N sampling was performed using a non-flexible flocked swab with normal tip (in Amies transport medium, Cat. number 480CE, Copan ${ }^{\circ}$ ). rRTPCR for SARS-CoV-2 RNA was performed on both the NP swab and the OP/N swab according to Corman et al. [15] using NucliSens easyMag ${ }^{\text {mat }}$ RNA extraction (bioMérieux, Marcy-l'Étoile, France) and one-step rRT-PCR (Qiagen One Step RT-PCR Kit, Cat. number 210212, Qiagen, Hilden, Germany) on CFX96 cyclers (Bio-Rad, Hercules, USA). All tests were performed in-hospital on a daily basis. A cycle threshold $(\mathrm{Ct})$ value below 50 for E-gene was interpreted as positive for SARS-CoV-2 RNA.

All clinical samples were tested for inhibition by adding $7.5 \mu \mathrm{l}$ of Diagenode RNA extraction and inhibition Real-Time PCR control (Cat. Number DECR-CY-L100, Diagenode $\mathrm{SA}^{\circ}$, Belgium).

Since retesting was not performed in the ambulant patient group, sensitivity of $\mathrm{OP} / \mathrm{N}$ and NP sampling was considered in hospitalized patients only.

Descriptive and relative frequencies were used to describe the distribution of cases. Since the data were paired, the Wilcoxon signed-rank test was used to assess differences between cycle threshold ( $\mathrm{Ct}$, considered as $\log 2$ transformed data of quantity viral RNA load) in NP and $\mathrm{OP} / \mathrm{N}$ specimens. The Kruskal Wallis test was used to assess the differences between cycle threshold among different groups of disease severity. The correlation 
between NP PCR Ct and OP/N PCR Ct was assessed using Spearman's rank correlation. Sensitivity was calculated by means of cross tabs. Specificity was found not to be relevant since not one false positive SARSCoV-2 PCR-result was found in this trial period (over hundred samples), in line with literature. [5] Due to the nature of this trial, assessing sensitivity without a negative control group, a calculation of the weighted kappa coefficient was not relevant. All analyses were performed using $\mathrm{IMB}^{\circ} \mathrm{SPSS}^{\circ}$ Statistics version 26.

The study was approved by the ethical review board Ghent University Hospital (BC-07662). The need for written informed consent was waived by the institutional review board.

\section{Acknowledgements}

Not applicable.

\section{Authors' contributions}

All authors have read and approved the manuscript. Conceptualization: TD, PDP, BV. Methodology: TD, PDP, BV. Software: TD. Validation: BV, JB, LC, SV, EP. Formal analysis : BV, JB, LC, SV, EP. Investigation: TD, PDP, BV, JB, LC, SV, EP, EVB, SC, TM Resources: TD, PDP, BV, JB, LC, SV, EP, EVB, SC, TM Data Curation: TD. Writing - original draft preparation: TD. Writing - review and editing: TD, PDP, BV, JB, LC, SV, EP, EVB, SC, TM, ILR, AA, FV. Visualization: TD. Supervision: PDP, BV. Project administration: TD.

\section{Funding}

None.

\section{Availability of data and materials}

The datasets used and analysed during the current study are available from the corresponding author on reasonable request.

\section{Ethics approval and consent to participate}

The study is approved by the ethical review board. To limit the impact of the study on diagnostic flow and patient care, the need for written informed consent was waived by the institutional review board.

\section{Consent for publication}

Not applicable.

\section{Competing interests}

The authors declare that they have no competing interests.

\section{Author details}

${ }^{1}$ Emergency Department, Ghent University Hospital, Ghent, Belgium. ${ }^{2}$ Department of Medical Microbiology, Ghent University Hospital, 9000 Ghent, Belgium. ${ }^{3}$ Department of Infection Control, Ghent University Hospital, Ghent, Belgium. ${ }^{4}$ Department of Internal Medicine \& Infectious diseases, Ghent University Hospital, Ghent, Belgium. ${ }^{5}$ Department of Respiratory Medicine, Ghent University Hospital, Ghent, Belgium. ${ }^{6}$ Department of Thoracic and Vascular Surgery, Chief Physician and Head of COVID-19 task force, Ghent University Hospital, Ghent, Belgium.

Received: 15 September 2020 Accepted: 10 January 2021

Published online: 22 January 2021

\section{References}

1. Zhu N, Zhang D, Wang W, et al. A novel coronavirus from patients with pneumonia in China. N Engl J Med. 2020;382:727-7733. DOl:https://doi.org/ 10.1056/NEJMoa2001017.

2. Wang D, Hu B, Hu C, et al. Clinical characteristics of 138 hospitalized patients with 2019 novel coronavirus-infected pneumonia in Wuhan. China JAMA. 2020;323:1061-9. DOl:https://doi.org/10.1001/jama.2020.1585.

3. World Health Organization. Laboratory testing for 2019 novel coronavirus (2019-nCoV) in suspected human cases. Interim guidance. 19 March 2020.
Accessed on April 29. 2020. https://www.who.int/publications-detail/ laboratory-testing-for-2019-novel-coronavirus-in-suspected-human-cases-202 00117.

4. Centers for Disease Control and prevention. Interim Guidelines for Collecting, Handling, and Testing Clinical Specimens from Persons for Coronavirus Disease 2019 (COVID-19). Accessed on April 29. 2020. https:// www.cdc.gov/coronavirus/2019-nCoV/lab/guidelines-clinical-specimens.html.

5. Péré H, Podglajen I, Wack M, et al. Nasal swab sampling for SARS-CoV-2: A convenient alternative in time of nasopharyngeal swab shortage. J Clin Microbiol. 2020;58:e00721-20. DOl:https://doi.org/10.1128/JCM.00721-20.

6. Azzi L, Carcano G, Gianfagna $F$, et al. Saliva is a reliable tool to detect SARS CoV-2. J Infect. 2020;81:e45-50. DOl:https://doi.org/10.1016/j.jinf.2020.04.005.

7. Lin C, Xiang J, Yan M, Li H, Huang S, Shen C. Comparison of throat swabs and sputum specimens for viral nucleic acid detection in 52 cases of novel coronavirus (SARS-CoV-2)-infected pneumonia (COVID-19). Clin Chem Lab Med. 2020;58:1089-94. DOl:https://doi.org/10.1515/cclm-2020-0187.

8. Wang W, Xu Y, Gao R, et al. Detection of SARS-CoV-2 in different types of clinical specimens. Research letter JAMA. 2020;323:1843-4. DOl:https://doi. org/10.1001/jama.2020.3786.

9. Zou L, Ruan F, Huang M, et al. SARS-CoV-2 viral load in upper respiratory specimens of infected patients. Letter to the editor. N Engl J Med. 2020;382: 1177-9. DOl:https://doi.org/10.1056/NEJMc2001737.

10. Yang $Y$, Yang $M$, Shen $C$, et al. Evaluating the accuracy of different respiratory specimens in the laboratory diagnosis and monitoring the viral shedding of 2019-nCoV infection. MedRXiv 2020; available from: https://doi. org/10.1101/2020.02.11.20021493.

11. Wang $X$, Tan L, Wang $X$, et al. Comparison of nasopharyngeal and oropharyngeal swabs for SARS-CoV-2 detection in 353 patients received tests with both specimens simultaneously. Int J Infect Dis. 2020;94:107-9. DOl:https://doi.org/10.1016/j.ijid.2020.04.023.

12. Vermeiren C, Marchand-Senécal X, Sheldrake E, et al. Comparison of Copan Eswab and FLOQswab for COVID-19 PCR diagnosis: working around a supply shortage. J Clin Microbiol. 2020;58:e00669-20. DOl:https://doi.org/10. 1128/JCM.00669-20

13. Cheng MP, Papenburg J, Desjardins M, et al. Diagnostic Testing for Severe Acute Respiratory Syndrome-Related Coronavirus-2: A Narrative Review. Ann Intern Med. 2020;172:726-34. DOl:https://doi.org/10.7326/M20-1301.

14. National Institutes of Health $(\mathrm{NIH})$. Clinical Presentation of People with SARS-CoV-2 Infection. Accessed on December 12. 2020. https://www.covid1 9treatmentguidelines.nih.gov/overview/clinical-presentation/.

15. Corman VM, Landt O, Kaiser M, et al. Detection of 2019 novel coronavirus (2019-nCoV) by real-time RT-PCR. Euro Surveill. 2020;25:23-31. DOl:https:// doi.org/10.2807/1560-7917.ES.2020.25.3.2000045

\section{Publisher's Note}

Springer Nature remains neutral with regard to jurisdictional claims in published maps and institutional affiliations.

Ready to submit your research? Choose BMC and benefit from

- fast, convenient online submission

- thorough peer review by experienced researchers in your field

- rapid publication on acceptance

- support for research data, including large and complex data types

- gold Open Access which fosters wider collaboration and increased citations

- maximum visibility for your research: over $100 \mathrm{M}$ website views per year

At BMC, research is always in progress.

Learn more biomedcentral.com/submission 